\title{
Alucinaciones y apariciones: Exploración intercultural de mediciones perceptuales entre estudiantes limeños y pucallpinos ${ }^{1}$
}

\author{
Alejandro Parra, Universidad Abierta Interamericana de Buenos Aires (Argentina) \\ Luis Espinoza Paul, Colegio "Coronel Portillo", Pucallpa, Ucayali (Perú) \\ Recibido: 5 de mayo del 2009 / Aprobado: 15 de julio del 2009
}

Se estudiaron dos muestras: una compuesta por 214 estudiantes de psicología de una universidad de Lima (74\% mujeres y 26\% varones), y la segunda por 184 estudiantes de educación secundaria de mayores de Pucallpa, (42\% mujeres y $58 \%$ varones). Tanto estudiantes limeños como pucallpinos con experiencias aparicionales mostraron mayor propensidad a experimentar percepciones inusuales pseudoalucinatorias que quienes no tuvieron la experiencia, así como también mayor propensión a la esquizotipia en comparación con quienes no tuvieron estas experiencias.

propensidad a alucinar / tendencias disociativas / propensidad a la fantasía / personalidad esquizotípica

\section{Hallucinations and apparitions: Intercultural exploration of perceptual measures between students from Lima and students from Pucallpa}

Two samples were studied, 214 students of psychology at a university in Lima (74\% women, 26\% men) and 184 upper secondary students in Pucallpa, Peruvian region of Ucayali (42\% women, $58 \%$ men). Results show that both students from Lima and from Pucallpa that had had aparitional experiences were more prone to experiment unusual pseudo-hallucinatory perceptions and more prone to skizotypy in comparison to those who reported not having experiences of the sort. In addition, students that had had aparitional experiences showehigher levels in the scales of disociation, absortion and proness to phantasy compared to students with no aparitional experiences.

hallucination proneness / dissociation / fantasy proneness /

cognitive perceptual schizotypy

Correos electrónicos: rapp@fibertel.com.ar; lespinoza@nipa-peru.com

1 Este estudio fue posible gracias al financiamiento de la Fundación BIAL de Portugal. Agradecemos también al Dr. William Acuña de la Facultad de Psicología de la Universidad Ricardo Palma de Lima, Perú, al licenciado Carlos Malpartida y Motecilo, del colegio "Coronel Portillo" de Pucallpa, Ucayali, y al licenciado Pedro Tapia, por su cooperación para la administración de los cuestionarios. 


\section{INTRODUCCIÓN}

Wallace (1959) se refirió a las alucinaciones como "uno de los modos más antiguos y ampliamente distribuidos de experiencia humana". Existe abundante literatura antropológica descriptiva de experiencias alucinatorias en cientos de sociedades de todo el mundo. La antropóloga Erika Bourguignon (1970) estudió muestras pertenecientes a 488 sociedades para examinar experiencias disociativas, o más ampliamente, estados alterados de conciencia.

También se han encontrado diferencias culturales e históricas en la tipología de las alucinaciones (Al-Issa, 1977). Por ejemplo, las alucinaciones visuales parecen ser más comunes en los países del Tercer Mundo que en los países desarrollados; observación esta que fue confirmada en el estudio multinacional de nuevos casos de psicosis de la Organización Mundial de la Salud (Sartorius, Shapiro \& Jablensky, 1974). Incluso parece haber una disminución de las experiencias alucinatorias visuales en Occidente en comparación con un aumento de las auditivas (Kroll \& Bachrach, 1982).

Thalbourne (1982) definió la "aparición" como una experiencia visual en la cual parece estar presente una persona o un animal (vivo o muerto) que está fuera del alcance sensorial de quien lo experimenta. Es difícil definir el término "aparición" sin introducir ciertas presunciones teóricas acerca de la naturaleza de las apariciones, sobre todo provenientes de sistemas de creencia en espíritus y otras fuerzas sobrenaturales.

Hay investigaciones (Bennett, 1987; Davidson \& Russel, 1981; Emmons, 1982; Finucane, 1984) que muestran cómo ha variado la experiencia a lo largo de la historia y en todos los grupos sociales de acuerdo con las funciones sociales de la creencia en apariciones. Desde una perspectiva transcultural, el sistema de creencias del individuo puede ayudar a determinar si este admite o no que las experiencias particulares son generadas por sí mismos o por otros (Al Issa, 1977, 1995). En la mayoría de las culturas occidentales, las alucinaciones tienden a ser consideradas como amenazantes, pero en las culturas no occidentales pueden considerarse como experiencias sagradas, lo cual corresponde a la distinción entre las interpretaciones psicológicas y las religiosas.

Por ejemplo, los análisis cuantitativos ponen en evidencia que la sensación de presencia es una experiencia común en la experiencia conocida como "parálisis del sueño" asociada a alucinaciones visuales, auditivas y táctiles, e intenso temor (Cheyne, NewbyClark \& Rueffer, 1999). La parálisis del sueño es un estado de inmovilidad involuntaria consciente que ocurre antes de dormir o inmediatamente antes de despertar. Un episodio puede durar desde pocos segundos hasta varios minutos, de hecho, aproximadamente el 
$30 \%$ de adultos jóvenes dijeron haber tenido experiencias de parálisis del sueño (Cheyne, Newby-Clark \& Rueffer, 1999; Fukuda et al., 1998; Spanos et al., 1995).

Hay poca información acerca del perfil de personalidad del individuo que experimenta apariciones. McCreery y Green (1986) encontraron puntajes de psicosis, neurosis y extraversión comparables a los de la población general, pero no hicieron una comparación con individuos sin experiencias aparicionales. Sin embargo, Irwin (1989) observó que la imaginería mental parece estar involucrada en la experiencia. Otros estudios mostraron que individuos con y sin experiencias aparicionales no difieren en su capacidad de absorción psicológica (Houg, 1991), aunque sí una marcada necesidad de procesar sus imágenes mentales (Irwin, 1985), lo cual es consistente con la observación de Palmer (1979) según la cual individuos que tienen experiencias aparicionales analizan sus propios sueños y practican meditación. La propensión a imaginar emerge como un fuerte discriminador: como grupo, estos individuos parecen estar inclinados a fantasear (Cameron \& Roll, 1983; Osis, 1986; Wilson \& Barber, 1983).

Las experiencias aparicionales se presentan con mayor frecuencia en individuos con bajo nivel educativo y en viudas o viudos; por ejemplo, la sensación de presencia es relativamente co- mún en personas que enviudaron recientemente. Este aspecto de "alucinación benigna" (de gente sana) es importante porque estas alucinaciones difieren de las que se observan en la enfermedad mental o por efecto de las drogas (Anderson \& Anderson, 1982; Asaad \& Shapiro, 1986).

Otros estudios también han demostrado (Mavromatis, 1987; Sherwood, 1999) que las apariciones ocurren a menudo en estados de conciencia en los cuales las imágenes mentales tienen la particularidad de parecer vivas y "reales" (por ejemplo, en los estados hipnagógico/hipnopómpico). Los pacientes psicóticos con alucinaciones auditivas difieren de los pacientes que no alucinan, y de las personas normales al tener un "testeo de realidad" debilitado (Slade, 1976; Johnson \& Raye, 1981). El testeo de realidad se refiere a la habilidad de distinguir una percepción actual (realidad) de un acto de imaginación actual (alucinación o aparición). El testeo de realidad también es importante para las personas normales que tienen alucinaciones visuales o apariciones, aunque los objetos son recuerdos evocados por la memoria de una aparición vista en el pasado, es decir que se refiere a la distinción entre una percepción pasada y un acto de imaginación pasado. En conclusión, el testeo de realidad es importante, pero un aspecto de este es la habilidad de focalizar la atención (absorción), y el otro es la habilidad de crear 
historias por fuera de la imaginería visual (propensión a la fantasía) del individuo.

Este estudio apunta a analizar si las experiencias aparicionales, la sensación de presencia — que se refiere a la vívida sensación de alguien o algo presente en un lugar (por ejemplo, una habitación) donde en realidad no hay nadie (Cheyne, Newby-Clark, \& Rueffer, 1999) - y la experiencia espontánea de mediumnidad/posesión — que se refiere a casos en los que el cuerpo de una persona es tomado por otra personalidad o entidad - son fenómenos dignos de estudio, como otros aspectos de la experiencia humana. De este modo, el enfoque está en la experiencia, cualquiera que sea la interpretación de sus experientes. Las apariciones, como todo en la vida mental de un individuo saludable, no ocurren en un vacío, sino que se interconecta con muchos otros procesos psicológicos. Nosotros replicamos una investigación anterior (Parra, 2008) sobre una muestra de estudiantes universitarios que midió una diversidad de experiencias perceptuales inusuales.

Sus resultados mostraron que aquellos individuos que habían tenido varias modalidades de experiencias aparicionales puntuaban más alto en escalas dimensionales de absorción, disociación, propensión a la fantasía y esquizotipia cognitivo-perceptual en comparación con quienes no tuvieron la experiencia.
Uno de nosotros (Espinoza Paul) realizó un estudio exploratorio mediante una encuesta on-line para relacionar la absorción psicológica y la incidencia de experiencias aparicionales en una muestra de 125 usuarios de internet peruanos.

Los resultados mostraron que el $55 \%$ de los encuestados reportó haber tenido al menos una vez en su vida una experiencia aparicional. También se encontró una correlación positiva con los reportes de experiencias aparicionales $($ Media $=20.49$; vs. Media $=$ $18.00 ; \mathrm{t}=-1.90, \mathrm{p}=.03$, a una cola), lo cual sugiere que las experiencias aparicionales pueden estar correlacionadas con procesos cognitivos similares a los de la absorción psicológica (Espinoza, 2004).

Para este estudio intercultural elegimos dos poblaciones: una muestra de estudiantes de psicología de la ciudad de Lima (población similar al estudio de Parra) y una muestra de estudiantes residentes en la ciudad de Pucallpa.

En Pucallpa conviven diversos grupos étnicos nativos como los cashibos, shipibos, conibos y shetebos, y forman parte de diferentes grupos étnicos, descendientes de la familia lingüística pano, tales como shipibos-conibos, shetebos y pisquibos.

En el 2007 (un año antes del presente estudio), la prensa pucallpina difundió la noticia de que al menos cinco estudiantes de la escuela Faustino Mal- 
donado habían experimentado convulsiones, supuestamente por participar en el llamado juego de la "Ouija".

Los estudiantes mostraron comportamientos extraños y proferían gritos e insultos, según el informe periodístico. A consecuencia de ello, las autoridades sanitarias iniciaron una campaña para orientar a padres de familia, profesores y escolares sobre "lo peligroso que es jugar a la Ouija" (http:// www.globovision.com/news.php?nid=65752).

Como no tuvimos acceso a esta escuela, uno de nosotros (Espinoza) obtuvo el permiso de dos escuelas secundarias de características demográficas similares para administrar los cuestionarios.

Pusimos a prueba cinco hipótesis específicas: que los estudiantes que ven apariciones o que tienen otras modalidades semejantes de experiencias tienen puntajes altos de 1) experiencias alucinatorias auditivas, visuales, táctiles e hipnagógico-hipnopómpicas; 2) propensidad a la esquizotipia (incluyendo el subfactor de E. cognitivo-perceptual); y 3) disociación, 4) absorción, 5) propensidad a la fantasía, en comparación con quienes no tienen tales experiencias.

Un estudio exploratorio ad hoc en este estudio es determinar posibles diferencias entre los estudiantes universitarios de Lima y los estudiantes secundarios de Pucallpa.

\section{MÉTOdo}

\section{Participantes}

Se estudiaron dos muestras: una compuesta por 214 estudiantes de ambos sexos de la Facultad de Psicología de la Universidad Ricardo Palma, 159 (74\%) mujeres y $55(26 \%)$ varones, con un rango etario de 17 a 47 años (Media $=$ 20.87; $\mathrm{SD}=3.51$ ) residentes en Lima y alrededores.

La segunda muestra estuvo compuesta por 184 estudiantes del Colegio de Educación Secundaria de Mayores "Coronel Portillo", en Pucallpa, Ucayali, 79 (42\%) mujeres y 107 (58\%) varones, con un rango etario de 17 a 50 años (Media $=19,09 ; \mathrm{SD}=4,53)$. Ningún estudiante, en ambas muestras, recibió compensación económica.

\section{Instrumentos}

Los estudiantes limeños completaron cinco escalas relacionadas con experiencias perceptuales inusuales: El Cuestionario de Alucinaciones (CEA), la Escala de Experiencias Disociativas (DES), el Cuestionario de Experiencias Creativas (CEQ), la Escala de Absorción de Tellegen (TAS) y el Cuestionario de Personalidad Esquizotípica (SPQ).

El Cuestionario de Alucinaciones (CEA) (Parra, Adróver \& González, 2006; alfa de Cronbach= .93), mediante 38 ítems, mide la propensidad a alu- 
cinar en seis modalidades sensoriales con una escala tipo Likert $0-5$, siendo 0 nunca a 5 frecuentemente. Solo se usaron cuatro modalidades: auditivo ("Cuando estoy completamente solo en casa, oigo una voz que me llama por mi nombre, una sola vez. Por ejemplo: Carmen); visual ("He visto sombras, o figuras humanas o no-humanas cerca de mi cama, yo las he visto claramente y veo lo que hacen"); táctil ("He tenido la experiencia de sentir una palmada en mi hombro, o cualquier otra sensación vívida de contacto fisico de otra persona detrás mío, pero cuando me doy vuelta no veo a nadie"); e hipnagógico/hipnopómpico ("Solo cuando me estoy durmiendo o estoy despertando del sueño, he oído voces o diálogos, música o melodías, sonidos, a veces agradables y otras no, pero que escucho nítidamente").

Escala de Experiencias Disociativas (DES) (Bernstein \& Putman, 1993; alfa de Cronbach $=.91)$, es una escala trifactorial de 28 ítems en una escala Likert $0-10$, que mide una variedad de tendencias disociativas: amnesia, despersonalización y desrealización (por ejemplo, "Hay personas que tienen la experiencia de conducir o de estar viajando en un automóvil, colectivo o microbús y de repente se dan cuenta de que no recuerdan lo que pasó durante todo o parte del viaje").

Cuestionario de Experiencias Creativas (CEQ) (Merckelbach, Horselenberg \& Muris, 2001; alfa de Cronbach
$=.89$ ), escala de 25 ítems de respuesta verdadero/falso, mide la propensidad a la fantasía, especialmente en la niñez (por ejemplo, "Cuando veo escenas de violencia por televisión, me siento tan involucrado en estas que me provoca mucha inquietud").

Escala de Absorción de Tellegen (TAS) (Tellegen \& Atkinson, 1974; alfa de Cronbach $=.91)$, mide mediante 34 ítems de respuesta verdadero/ falso la frecuencia con que una persona se involucra en actividades que exigen atención y concentración (por ejemplo, "Cuando escucho música de órgano u otra música imponente a veces siento como si me estuvieran levantando en el aire").

Se empleó también una medida de rasgos de personalidad: el Cuestionario de Personalidad Esquizotípica (SPQ) (Raine, 1991; Raine, 1992, Raine \& Baker, 1992; Raine \& Benishay, 1995; alfa de Cronbach = .91), una escala trifactorial de 74 ítems de respuesta dicotómica si/no, que mide estilos de pensamiento esquizotípicos (se empleó la puntuación total del SPQ y el subfactor 1 Cognitivo perceptual; por ejemplo: "¿Alguna vez ha visto cosas que para los demás son invisibles?" o "¿Son sus pensamientos a veces tan fuertes que casi podría escucharlos?").

Respecto a las experiencias aparicionales, sensación de presencia y mediumnidad, se formularon tres preguntas (basadas en la encuesta de Palmer, 1979): una para Experiencia Aparicio- 
nal (EA) - ("Estando despierto, he tenido la experiencia de oír voces o ver presencias invisibles para otros que me indicaban acerca de un peligro inminente que luego ocurrió"); la segunda para Sensación de Presencia (SP) ("Estando solo, he tenido la vívida impresión de una sensación de presencia, pero no visible donde me encontraba"); y la tercera para Mediumnidad (M) ("He tenido la experiencia de sentir que mi cuerpo no me pertenecía y que estaba siendo controlado por otra fuerza aparte de mí").

\section{Procedimiento}

Aplicamos una técnica de muestreo no probabilística para obtener el mayor número de casos para analizar. El set de los test autoadministrables fue entregado en un sobre A4 a cada estudiante, en forma contrabalanceada, durante una clase. El tiempo promedio para completar los cuestionarios fue de 50 minutos (30 minutos para el grupo de Pucallpa).

Los estudiantes recibieron una vaga información del objeto de estudio y se les invitó a participar voluntaria y anónimamente completando los test, en una única sesión, en días y horarios previamente pactados con los docentes.
El orden de administración de ambas pruebas fue contrabalanceado y los cuestionarios de alucinaciones se presentaron bajo el seudotítulo de Cuestionario de Experiencias Psicológicas, con lo cual se evitó sesgar las respuestas.

Por razones de tiempo y disponibilidad, el grupo de Pucallpa solo completó dos cuestionarios: el de Alucinaciones y el de Personalidad Esquizotípica, y aparte las tres preguntas de experiencias aparicionales.

Los análisis fueron procesados mediante el SPSS 11.5 (en español) y los análisis estadísticos fueron evaluados a dos colas. A causa de la alta asimetría en los puntajes de las escalas de la muestra, en lugar de un test t, aplicamos una $U$ de Mann-Whitney.

\section{Resultados}

Sobre la base de las respuestas obtenidas para las preguntas de experiencia aparicional (EA, SP y M) se conformaron dos grupos para cada experiencia. Para agrupar a quienes tenían la experiencia aparicional se convirtieron las respuestas 1 (rara vez), 2 (ocasionalmente), 3 (a menudo) y 4 (muy frecuentemente). Para agrupar a quienes no tuvieron la experiencia se empleó solo la respuesta "Nunca". 
Tabla 1

Porcentaje de encuestados en varias categorías demográficas

\begin{tabular}{lcc}
\hline & Pucallpinos & Limeños \\
\hline Estado civil & & \\
Soltero & $177(95,2)$ & $208(97,2)$ \\
Casado & $7(3,8)$ & $6(2,8)$ \\
Separado/divorciado & $2(1,1)$ & - \\
Orden de nacimiento & & \\
$\quad$ Hijo único & $21(11,3)$ & $15(7,0)$ \\
Primer nacimiento & $34(18,3)$ & $74(34,6)$ \\
Segundo nacimiento & $65(34,9)$ & $59(27,6)$ \\
Último hermano & $65(34,9)$ & $66(30,8)$ \\
Religión & & \\
Católico & $133(71,5)$ & $171(79,9)$ \\
Judío & - & $1(, 5)$ \\
Cristiano no denominacional & $41(22,0)$ & $9(4,2)$ \\
No denominacional & $1(0,5)$ & $12(5,6)$ \\
Filosofías orientales & $5(2,7)$ & $3(1,4)$ \\
Ateos & $6(3,2)$ & $18(8,4)$ \\
Pensamiento político & & \\
$\quad$ Muy conservador & $50(26,9)$ & $3(1,4)$ \\
Conservador & $59(31,7)$ & $24(11,2)$ \\
Moderado & $15(8,1)$ & $117(54,7)$ \\
Liberal & $44(23,7)$ & $65(30,4)$ \\
Muy liberal & $18(9,7)$ & $5(2,3)$ \\
Espiritualidad & & $11(5,1)$ \\
No soy espiritual & $45(24,2)$ & $71(33,2)$ \\
Soy un poco espiritual & $82(44,1)$ & $97(45,3)$ \\
Soy moderadamente espiritual & $30(16,1)$ & $3(1,4)$ \\
Soy muy espiritual & $22(11,8)$ & $7(3,8)$ \\
$\quad$ Soy extremadamente espiritual & &
\end{tabular}

Tabla 2

Experiencia aparicional, sensación de presencia y experiencias de mediumnidad

\begin{tabular}{llcc}
\hline Tipo de experiencia & Grupo & Limeños & Pucallpinos \\
\hline Aparicional & Sin experiencia & $173(80,8)$ & $106(57,0)$ \\
& Con experiencia & $\mathbf{4 1 ( 1 9 , 2 )}$ & $\mathbf{8 0}(\mathbf{4 3 , 0 )}$ \\
Sensación de presencia & Sin experiencia & $117(54,7)$ & $99(53,2)$ \\
& Con experiencia & $\mathbf{9 7 ( 4 5 , 3 )}$ & $\mathbf{8 7}(\mathbf{4 6 , 8 )}$ \\
Posesión & Sin experiencia & $186(86,9)$ & $124(66,7)$ \\
& Con experiencia & $\mathbf{2 8 ( 1 3 , 1 )}$ & $\mathbf{6 2 ( 3 3 , 3 )}$ \\
\hline
\end{tabular}


Tabla 3

Comparación de medias y SD entre estudiantes limeños $(n=214)$ y pucallpinos $(n=186)$ en las subescalas del Cuestionario de Personalidad Esquizotípica

\begin{tabular}{|c|c|c|c|c|}
\hline SPQ & Grupo & Media & SD & $\mathbf{t}$ \\
\hline \multirow[t]{2}{*}{ Ideas de referencia } & Limeños & 3,51 & 2,41 & $-1,10$ \\
\hline & Pucallpinos & 3,77 & 2,38 & \\
\hline \multirow[t]{2}{*}{ Ansiedad social } & Limeños & 3,29 & 2,32 & 1,06 \\
\hline & Pucallpinos & 3,06 & 1,94 & \\
\hline \multirow[t]{2}{*}{ Creencias bizarras } & Limeños & 2,22 & 1,87 &,- 69 \\
\hline & Pucallpinos & 2,34 & 1,50 & \\
\hline \multirow[t]{2}{*}{ Experiencias perceptuales inusuales } & Limeños & 2,73 & 2,02 &,$- 38^{*}$ \\
\hline & Pucallpinos & 2,81 & 1,70 & \\
\hline \multirow[t]{2}{*}{ Comportamiento excéntrico } & Limeños & 1,88 & 2,17 & $2,95^{\star *}$ \\
\hline & Pucallpinos & 1,37 & 1,00 & \\
\hline \multirow[t]{2}{*}{ Sin amigos cercanos } & Limeños & 2,09 & 1,55 & $10,81^{* * *}$ \\
\hline & Pucallpinos &, 78 & ,62 & \\
\hline \multirow[t]{2}{*}{ Discurso incoherente } & Limeños & 4,09 & 2,28 & $9,48^{* * *}$ \\
\hline & Pucallpinos & 2,27 & 1,36 & \\
\hline \multirow[t]{2}{*}{ Aplanamiento afectivo } & Limeños & 1,73 & 1,73 & $2,69^{\star * *}$ \\
\hline & Pucallpinos & 1,34 & ,94 & \\
\hline \multirow[t]{2}{*}{ Suspicacia } & Limeños & 9,37 & 5,75 & $19,56^{\star * *}$ \\
\hline & Pucallpinos & 1,04 & ,83 & \\
\hline \multirow[t]{2}{*}{ SPQ (puntaje total) } & Limeños & 30,92 & 11,51 & $8,79^{\star * *}$ \\
\hline & Pucallpinos & 21,57 & 8,17 & \\
\hline \multirow[t]{2}{*}{ Factor 1 E. Cognitivo-perceptual } & Limeños & 8,46 & 4,87 & $-3,61^{* * *}$ \\
\hline & Pucallpinos & 10,24 & 4,52 & \\
\hline \multirow[t]{2}{*}{ Factor 2 E. Interpersonal } & Limeños & 7,11 & 4,73 & $2,88^{\star * *}$ \\
\hline & Pucallpinos & 5,95 & 2,26 & \\
\hline \multirow[t]{2}{*}{ Factor 3 E. Desorganizada } & Limeños & 5,97 & 3,75 & $5,55^{\star * *}$ \\
\hline & Pucallpinos & 4,18 & 1,91 & \\
\hline
\end{tabular}

${ }^{*} p<.05 ;{ }^{* *} p<.01 ;{ }^{* * *} p<.001$ (todos los valores de $p$ a dos colas); $g l=398$ 

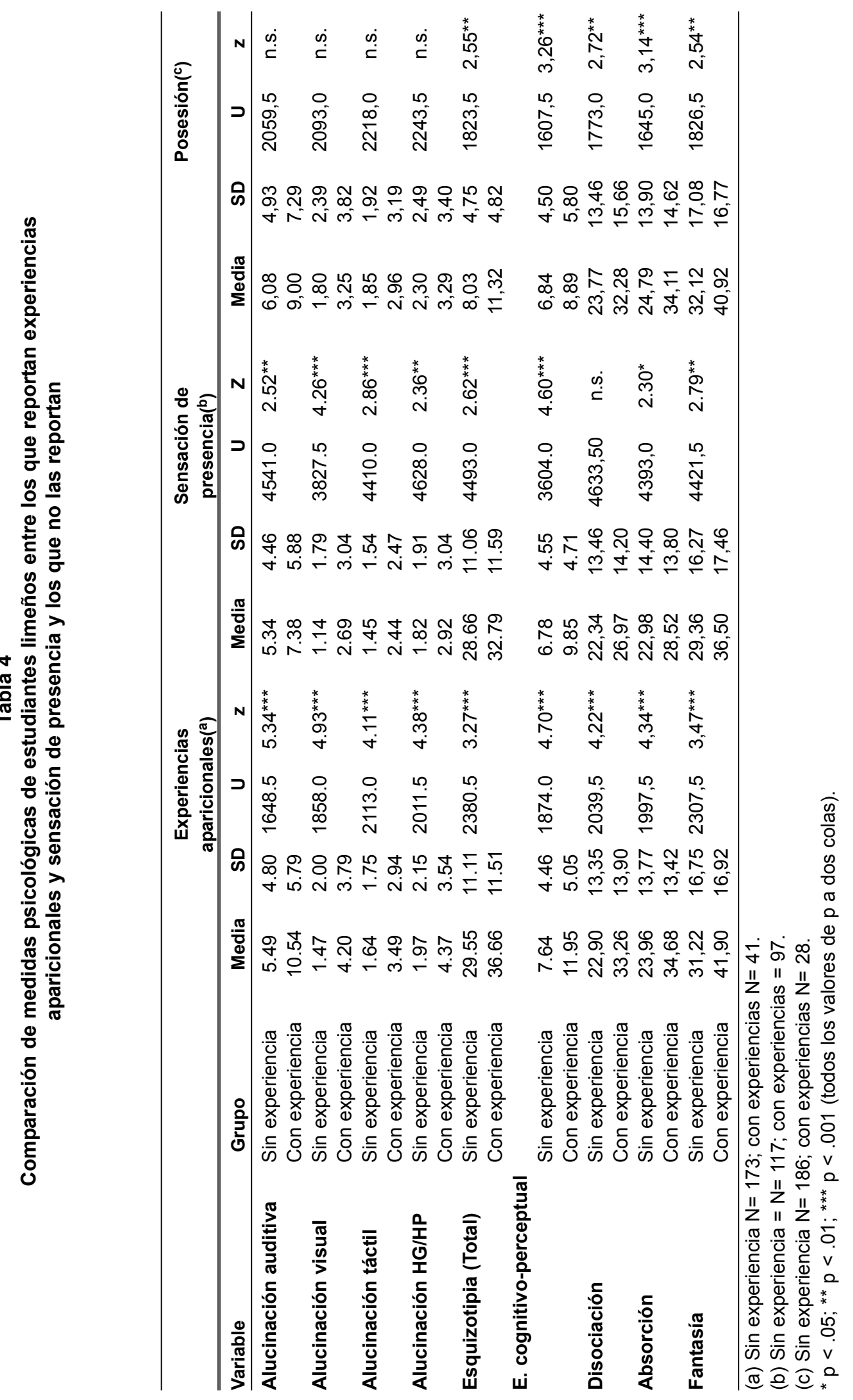


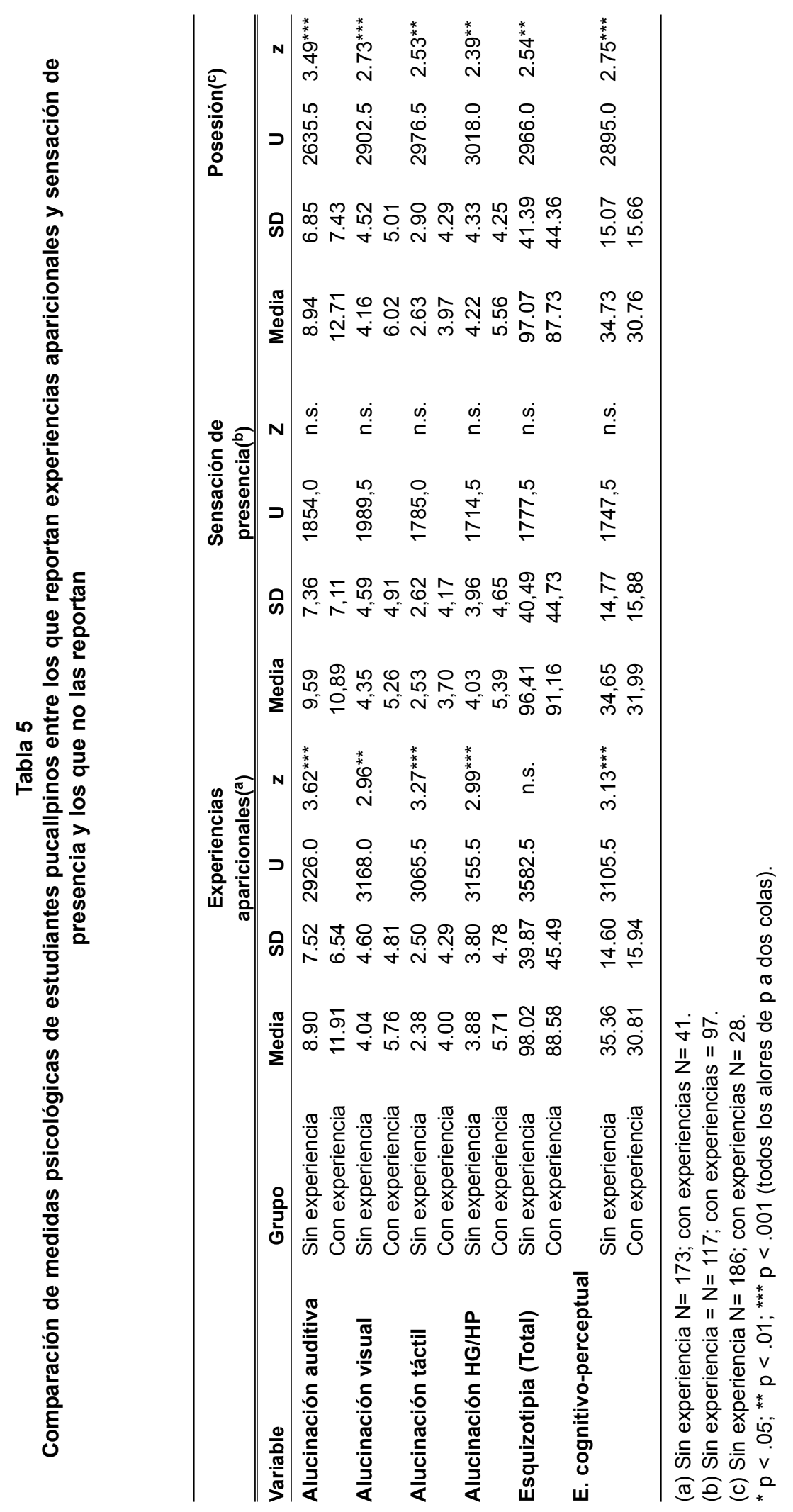




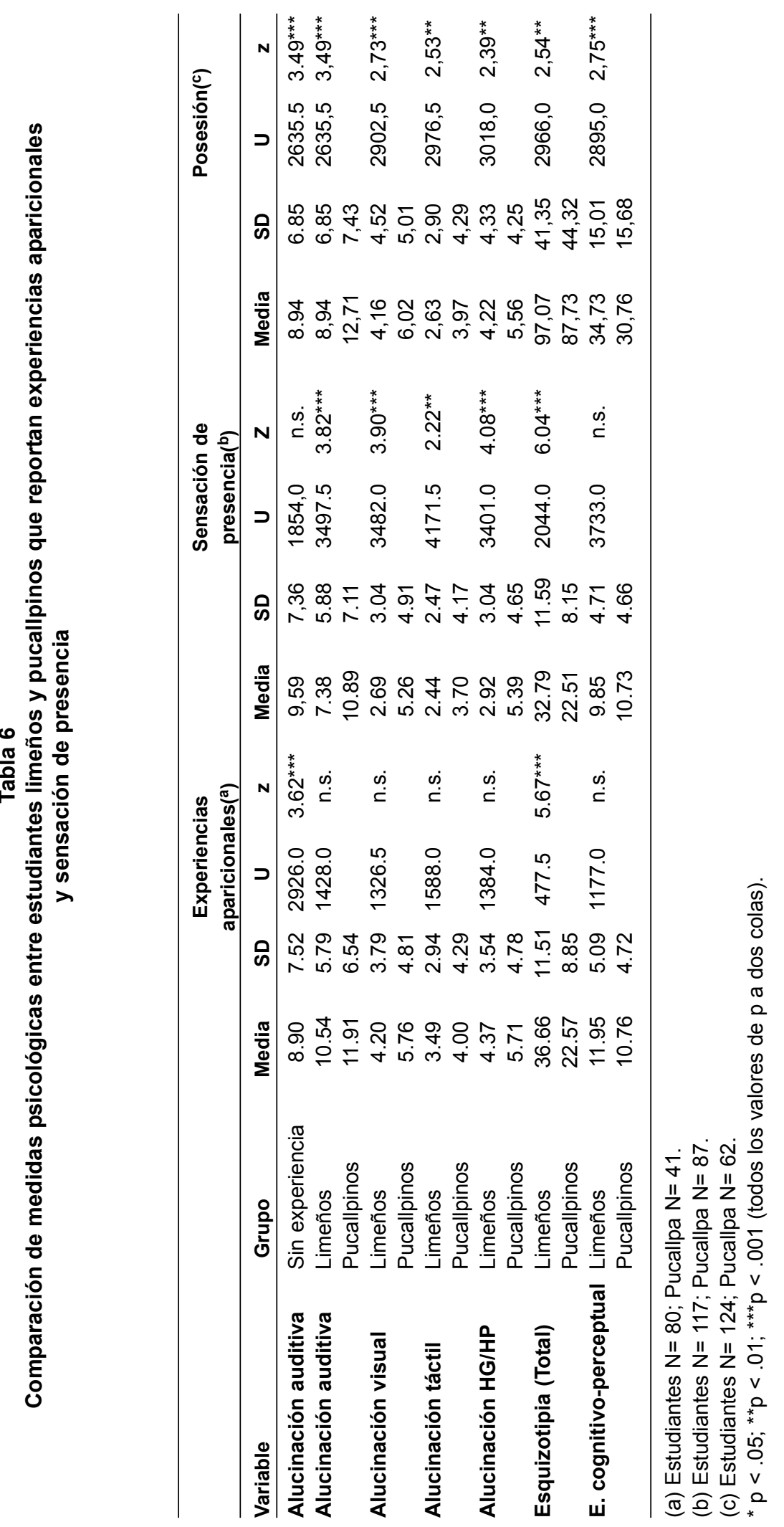




\section{Resultado de las hipótesis}

- Experiencias alucinatorias.- La hipótesis 1 es que los estudiantes limeños con experiencias aparicionales puntuarían más alto en alucinación (auditiva, visual, táctil y HG/HP) que quienes no tuvieron la experiencia, lo cual se confirmó para alucinación de modalidad auditiva (EA: $\mathrm{z}=5.34, \mathrm{p}<.001, \mathrm{a}$ dos colas; SP: $\mathrm{z}=2.52, \mathrm{p}<.001, \mathrm{a}$ dos colas), visual (EA: $\mathrm{z}=4.93, \mathrm{p}<.001$, a dos colas; SP: $\mathrm{z}=4.26, \mathrm{p}<.001$ ), táctil (EA: $\mathrm{z}=4.11, \mathrm{p}<.001$, a dos colas; SP: $\mathrm{z}=2.86, \mathrm{p}<.001$, a dos colas), e hipnagógico / hipnopómpica (EA: $\mathrm{z}=4.38, \mathrm{p}<.001$, a dos colas; SP: $\mathrm{z}=2.36, \mathrm{p}<.001, \mathrm{a}$ dos colas), donde la puntuación de alucinación para quienes tuvieron la experiencia aparicional era significativamente más alta que quienes no tuvieron la experiencia (véase la tabla 4), excepto mediumnidad, cuyos resultados no fueron significativos.

Asimismo, esta misma hipótesis señalaba que los estudiantes pucallpinos con experiencias aparicionales puntuarían más alto en alucinación (auditiva, visual, táctil y HG/HP) que quienes no tuvieron la experiencia, lo cual se confirmó para alucinación de modalidad auditiva $(E A: z=3.62, p<.001$, a dos colas; $\mathrm{M}: \mathrm{z}=3.49, \mathrm{p}<.001$, a dos colas), visual (EA: $\mathrm{z}=2.96, \mathrm{p}<.001$, a dos colas; $\mathrm{M}: \mathrm{z}=2.73, \mathrm{p}<.001$ ), táctil (EA: $\mathrm{z}=3.27, \mathrm{p}<.001$, a dos colas; M: $\mathrm{z}=2.53, \mathrm{p}<.001$, a dos colas), e hipnagógico / hipnopómpica (EA: $z=2.99, p<.001$, a dos colas; $\mathrm{M}: \mathrm{z}=2.39, \mathrm{p}<.001$, a dos colas), donde la puntuación de alucinación para quienes tuvieron la experiencia aparicional fue significativamente más alta que quienes no tuvieron la experiencia (véase la tabla 5), excepto sensación de presencia, cuyos resultados no fueron significativos.

- Propensión de esquizotipia.- La hipótesis 2 era que los estudiantes limeños con experiencias puntuarían más alto que quienes no tuvieron experiencias en propensión a la esquizotipia (medido con el SPQ-A), lo cual se confirmó (EA $\mathrm{z}=3.27$, $\mathrm{p}<.001$, a dos colas; SP: $\mathrm{z}=2.62$, $\mathrm{p}<.001$, a dos colas; y $\mathrm{M}=2.55$, $\mathrm{p}<.01$, a dos colas), donde la puntuación de esquizotipia para quienes tuvieron la experiencia aparicional era significativamente más alta que quienes no tuvieron la experiencia (véase la tabla 4). La hipótesis 2 era que los estudiantes de psicología con experiencias puntuarían más alto que quienes no tuvieron experiencias en esquizotipia cognitivo-perceptual, lo cual se confirmó (EA: $\mathrm{z}=3.27$, $\mathrm{p}<.001$, a dos colas; SP: $\mathrm{z}=2.62$, $\mathrm{p}<.001$, a dos colas y $\mathrm{M}: \mathrm{z}=2.55$, $\mathrm{p}<.01$, a dos colas) (véase la tabla 3), donde la puntuación de la modalidad cognitivo-perceptual para quie- 
nes tuvieron la experiencia aparicional fue significativamente más alta que quienes no tuvieron la experiencia (véase la tabla 4).

Igualmente, la hipótesis 2 fue que los estudiantes pucallpinos con experiencias puntuarían más alto que quienes no tuvieron experiencias en propensión a la esquizotipia, la cual fue confirmada (EA $z=3.13$, $\mathrm{p}<.001$, a dos colas; y $\mathrm{M}=2.75$, $\mathrm{p}<.001$, a dos colas), donde la puntuación de esquizotipia para quienes tuvieron la experiencia aparicional era significativamente más alta que quienes no tuvieron la experiencia (véase la tabla 5). La hipótesis 2 fue que los estudiantes pucallpinos con experiencias puntuarían más alto que quienes no tuvieron experiencias en esquizotipia cognitivo-perceptual (EA: $\mathrm{z}=3.13, \mathrm{p}<.001$, a dos colas; y M: $\mathrm{z}=2.75, \mathrm{p}<.01$, a dos colas) (véase la tabla 5), donde la puntuación de alucinación para quienes tuvieron la experiencia aparicional era significativamente más alta que quienes no tuvieron la experiencia (véase la tabla 5). En ambas comparaciones, sensación de presencia no obtuvo diferencia significativa.

- Disociación, absorción y propensión a la fantasía.- La hipótesis 3 fue que los estudiantes limeños con experiencias puntuarían más alto que quienes no tuvieron experiencias en disociación (medido con el
DES), la cual fue confirmada: quienes tienen experiencias aparicionales puntuaron significativamente más alto que quienes no la tuvieron (EA: $\mathrm{z}=4.22, \mathrm{p}<.001$, a dos colas; y $\mathrm{M}: \mathrm{z}=2.72, \mathrm{p}<.01$, a dos colas), excepto en sensación de presencia. La hipótesis 4 fue que los estudiantes con experiencias puntuarían más alto que quienes no la tuvieron en absorción (medido con el TAS), la cual fue confirmada: quienes tenían experiencias aparicionales puntuaron significativamente más alto que quienes no la tuvieron (EA: $z=4.34$, $\mathrm{p}<.001$, a dos colas; SP: $\mathrm{z}=2.30$, $\mathrm{p}<.05$, a dos colas y M: $\mathrm{z}=3.14$, $\mathrm{p}<.001$, a dos colas). La hipótesis 5 fue que los estudiantes con experiencias puntuarían más alto que quienes no tuvieron la experiencia en propensión a la fantasía (medido con el CEQ), la cual fue confirmada (EA: $\mathrm{z}=3.47, \mathrm{p}<.001$, a dos colas; SP $\mathrm{z}=2.79, \mathrm{p}<.001$, a dos colas; $\mathrm{y}$ $\mathrm{M}: \mathrm{z}=2.54 ; \mathrm{p}<.01, \mathrm{a}$ dos colas) (véase la tabla 4 ).

\section{Otros análisis}

Comparando a los estudiantes limeños con los estudiantes pucallpinos, estos últimos puntuaron significativamente más alto que los limeños en alucinación auditiva (SP: $\mathrm{z}=3.82, \mathrm{p}<.001$, a dos colas, y M: $\mathrm{z}=3,49, \mathrm{p}<.001$, a dos colas), visual (SP: $\mathrm{z}=3.90, \mathrm{p}<.001$, a dos colas; $y \mathrm{M}: \mathrm{z}=2.73, \mathrm{p}<.001$, a dos colas), táctil (SP: $\mathrm{z}=2.22, \mathrm{p}=.01$, a 
dos colas; $\mathrm{M}: \mathrm{z}=2.53, \mathrm{p}=.01$, a dos colas), y HG/HP (SP: z $=4.08$, $\mathrm{p}<.001$, a dos colas; $\mathrm{M}: \mathrm{z}=2.39$, $\mathrm{p}=.01$, a dos colas), excepto experiencia aparicional para todas las modalidades sensoriales de alucinación (véase la tabla 6).

Además, cuando se comparan ambas muestras de estudiantes, los pucallpinos puntuaron significativamente más alto que los estudiantes limeños en propensidad a la esquizotipia (EA: $\mathrm{z}=5.67, \mathrm{p}<.001, \mathrm{a}$ dos colas; SP: $\mathrm{z}=6.04, \mathrm{p}<.001$, a dos colas $\mathrm{y} \mathrm{M}$ : $\mathrm{z}=2.54, \mathrm{p}=.01$, a dos colas), y esquizotipia cognitivo-perceptual (M: $\mathrm{z}=2.75, \mathrm{p}=.01$, a dos colas), excepto experiencia aparicional y sensación de presencia (véase la tabla 6).

\section{DISCUSIÓN}

Los porcentajes de experiencias aparicionales son notables en ambos grupos, pero son significativamente superiores en los estudiantes pucallpinos (43\% y $33 \%)$ en comparación con los limeños (19\% y $13 \%$ ), cuyos valores se aproximan al $10 \%$ y $45 \%$ del estudio anterior de Parra (2008). Esto cambia para sensación de presencia, donde estudiantes limeños y pucallpinos tienen valores semejantes $(45,3 \%$ y $46,8 \%$, respectivamente) (en el estudio de Parra se obtuvo 9\%). Los resultados de este estudio también son muy similares al de Parra. Una comparación estadística entre la muestra de estudiantes de Parra
$(\mathrm{N}=656)$ con la del presente estudio reveló que aunque los estudiantes limeños puntuaron significativamente alto solo en propensidad a la esquizotipia para sensación de presencia $(\mathrm{z}=5.05$; $\mathrm{p}<.001$, a una cola), el resto de las mediciones psicológicas no mostraron diferencias significativas, y es posible concluir que ambas muestras guardan semejanzas en términos psicológicos.

Los estudiantes limeños con experiencias aparicionales mostraron mayor propensidad a experimentar percepciones inusuales seudoalucinatorias en las modalidades auditiva, visual, táctil e hipnagógico/hipnopómpica, excepto para mediumnidad, cuyos resultados no fueron significativos. De igual modo, los estudiantes pucallpinos puntuaron más alto en alucinación (auditiva, visual, táctil y HG/HP) en comparación con los que no tuvieron la experiencia, pero la modalidad aparicional cuyo resultado no fue significativo es sensación de presencia. Los estudiantes limeños y los pucallpinos con experiencias aparicionales también mostraron mayor propensión a la esquizotipia en comparación con quienes no tuvieron experiencias. Respecto a Disociación, Absorción y Propensión a la Fantasía: los estudiantes limeños que tienen experiencias aparicionales mostraron altos niveles en estas escalas en comparación con quienes no las tienen.

Los estudiantes pucallpinos mostraron mayores niveles de propensidad a alucinar en la modalidades auditiva, vi- 
sual, táctil e hipnagógico/hipnopómpica, así como también propensión a la esquizotipia. Estos indicadores de psicopatología son, en parte, coincidentes con el reporte del Instituto Especializado de Salud Mental (2005) evaluados con el Mini-International Neuropsychiatric Interview ${ }^{2}$ (Sheehan et al., 1998). Los resultados de este informe indican la incidencia en trastornos psiquiátricos de la población de Pucallpa, tales como valores altos en depresión $(21,4 \%)$ y ansiedad $(8,2 \%)$. Los trastornos psicóticos son realmente bajos en comparación $(0.2 \%)$. De acuerdo con este reporte, "en la selva peruana, la influencia cultural y su interrelación con el nivel educativo son especialmente importantes en problemas de salud, debido a que los mitos y prejuicios están muy extendidos y la medicina folclórica podría jugar un importante papel. Los estudios sobre el acceso a los servicios de salud mental sostienen que este se ve afectado principalmente en personas de menores recursos económicos y culturales, y lingüísticamente diferenciadas y con niveles educativos bajos" (Instituto Especializado de Salud Mental, 2005, p. 34).

Estos resultados también sugieren que las visiones de fantasmas se pueden relacionar con procesos cognitivos

2 El MINI tiene varios ítems sobre experiencias alucinatorias, pero no tuvimos acceso a esa base de datos. que involucran la propensión a la fantasía y la propensión a la esquizotipia cognitiva-perceptual, y que estos factores están correlacionados entre sí. Se puede concluir que las experiencias aparicionales están relacionadas con niveles altos de absorción, disociación y experiencias de fantasía/imaginación, lo cual coincide conceptualmente con los estudios que han encontrado que la propensión a la fantasía parece ser un predictor en otras experiencias espirituales (Myers, Austrin, Grisso \& Nickeson, 1983; Wilson \& Barber, 1983). La absorción es la capacidad de centrar la atención exclusivamente en algún objeto (incluyendo la imaginería mental), que parecen tener una elevada percepción de realidad, como ocurre con las apariciones (Espinoza, 2004; MacKenzie, 1982).

En segundo lugar, aunque hay una clara superposición entre las creencias paranormales, las experiencias paranormales y la esquizotipia, esto no significa necesariamente que las creencias y las experiencias paranormales estén asociadas al malestar psicológico (McCreery \& Claridge, 1986, 1995). McCreery y Claridge (2002: 144) denominaron al conjunto de estos síntomas positivos como "esquizotipia feliz", el cual representa un modelo cuasidimensional de esquizotipia que sugiere una extensión del modelo dimensional con la salud como punto de partida (Claridge, 1997; Claridge \& Beech, 1995). 
Debemos enfatizar que estamos tratando con afirmaciones que dependen solamente de respuestas de encuestas. El significado de estas afirmaciones no permite saber si las respuestas de los participantes están relacionadas con lo que preguntamos a menos que tengamos una descripción por escrito de tales experiencias perceptuales anómalas.

\section{REFERENCIAS}

Al-Issa, I. (1977). Social and cultural aspects of hallucinations. Psychological Bulletin, 84, 570-587.

Al-Issa, I. (1995). The illusion of reality or the reality of an illusion: Hallucinations and culture. British Journal of Psychiatry, 166, 368373.

Anderson, R. \& Anderson, W. (1982). Veridical and psychopathic hallucinations: A comparison of types. Parapsychology Review, 13(3), 17-23.

Asaad, G. \& Shapiro, B. (1986). Hallucinations: Theoretical and clinical overview. American Journal of Psychiatry, 143, 1088-1097.

Bennett, G. (1987). Traditions of belief: Women and the supernatural. Londres: Penguin Books.

Bernstein, E. M. \& Putnam, F. W. (1986). Development, reliability, and validity of a dissociation scale.
Journal of Nervous and Mental Disease, 174, 727-735.

Bourguignon, E. (1970). Hallucinations and trance: An anthropologist's perspective. En W. Keup (Ed.). Origins and mechanisms of hallucinations (pp. 83-90). Nueva York: Plenum.

Cameron, T. \& Roll, W. G. (1983). An investigation of apparitional experiences. Theta, 11, 74-78.

Claridge, G. (1997). Theoretical background and issues. En G. Claridge (Ed). Schizotypy: Implications for Illness and Health (pp. 3-18). Oxford: Oxford University Press.

Claridge, G. \& Beech, T. (1995). Fully and quasi-dimensional constructions of schizotypy. En A. Raine; T. Lencz \& S. A. Mednick (Eds.). Schizotypal Personality (pp. 192216). Cambridge: Cambridge University Press.

Cheyne, J. A.; Newby-Clark, I. R. \& Rueffer, S. D. (1999). Sleep paralysis and associated hypnagogic and hypnopompic experiences'. Journal of Sleep Research, 8, 313-317.

Davidson, H. R. E. \& Russell, W. M. S. (Eds.) (1981). The folklore of ghosts. Cambridge, Inglaterra: Brewer.

Espinoza, L. (2004). Experiencias aparicionales y absorción psicológica: 
Un estudio exploratorio on line. En F. E. da Silva (Ed.). Segundo Encontro Psi: Refletindo sobre o Futuro da Parapsicología (pp. 76-81). Curitiba, Paraná: Facultades Integradas "Espírita."

Emmons, C. F. (1982). Chinese ghosts and ESP. A study of paranormal beliefs and experiences. Metuchen, Nueva Jersey: Scarecrow Press.

Finucane, R. C. (1984). Appearances of the dead: A cultural history of ghosts. Buffalo, Nueva York: Prometheus Books.

Fukuda, K.; Ogilvie, R. D.; Chilcott, L.; Vendittelli, A. M. \& Takeuchi, T. (1998). The prevalence of sleep paralysis among Canadian and Japanese college students. Dreaming, 8, 59-66.

Hough, M. (1991). Cognition and the apparitional experience. Australian Parapsychological Review, 17, 8-16.

Instituto Especializado de Salud Mental (2005). Estudio Epidemiológico en Salud Mental en la Selva Peruana 2004: Informe general. Anales de Salud Mental, 21(1-2), 1-214.

Irwin, H. J. (1985). Parapsychological phenomena and the absorption domain. Journal of the American Society of Psychical Research, 79, $1-11$.
Irwin, H. J. (1989). Extrasensory experiences and the need for absorption. Parapsychology Review, 20(6), 9-10.

Johnson, M. \& Raye, C. (1981). Reality monitoring. Psychological Review, 88, 6785 .

Kroll, J. \& Bachrach, B. (1982). Visions and psychopathology in the Middle Ages. Journal of Nervous and Mental Disease, 170, 41-49.

MacKenzie, A. (1982). Hauntings and apparitions. Londres: Paladin.

Mavromatis, A. (1987). Hypnagogia: The unique state of consciousness between wakefulness and sleep. Londres: Routledge \& Kegan Paul.

McCreery, C. \& Green, C. (1986). A follo-up study of people reporting apparitional experiences. Informe presentado en la Décima Conferencia Internacional de la Society for Psychical Research, en Cambridge.

McCreery, C. \& Claridge, G. (1995). Out-of-the-body experiences and personality. Journal of the Society for Psychical Research, 60, 129148.

McCreery, C. \& Claridge, G. (2002). Healthy schizotypy: The case of out-of-the-body experiences. Personality and Individual Differences, 32, 141-154. 
Merckelbach, H.; Horselenberg, R. \& Muris, Peter (2001). The Creative Experiences Questionnaire (CEQ): A brief self-report measure of fantasy proneness. Personality and Individual Differences, 31, 987-995.

Myers, S. y Austrin, H. (1985). Distal eidetic technology. Journal of Mental Imagery, 9, 5766.

Osis, K. (1986). Apparitions old and new. En K. R. Rao (Ed.). Case studies in parapsychology (pp. 74-86). Jefferson, NC: McFarland.

Palmer, J. (1979). A community mail survey of psychic experiences. Journal of the American Society for Psychical Research, 81, 111-123.

Parra, A. (2008). Medidas psicológicas en relación con experiencias alucinatorias y experiencias aparicionales. Persona, 11, 109-128.

Parra, A.; Adróver, F. \& González, G. (2006). Estudio exploratorio de la experiencia alucinatoria: Comparación entre población clínica y noclínica. En A. Trimboli; J. C. Fantin; S. Raggi \& P. Fridman (Eds.). Encrucijadas actuales en salud mental: Primer Congreso Argentino de Salud Mental (pp. 258-267). Buenos Aires: Akadia.

Raine, A. (1991). The SPQ: A scale for the assessment of schizotypal personality based on DSM-III-R criteria. Schizophrenia Bulletin, 17, 556564.
Raine, A. (1992). Sex differences in schizotypal personality in a non-clinical population. Journal of $A b-$ normal Psychology, 101, 361-364.

Raine, A. \& Baker, L. (1992). The Schizotypal Personality Questionnaire: Genetics, Psychophysiology. Neuropsychology and Gender Differences. Western Psychological Association, Portland, Oregon, abril 30-mayo 3.

Raine, A. \& Benishay, D. (1995). The SPQ-B: A brief screening instrument for schizotypal personality disorder. Journal of Personality Disorders, 9, 346-355.

Sartorius, N.; Shapiro, R. \& Jablensky, A. (1974). The international pilot study of schizophrenia. Schizophrenia Bulletin, 1, 2125.

Sheehan, D. V.; Lecrubier, Y.; Sheehan, K. H.; Amorim, P.; Janavs, J.; Weiller, E.; Hergueta, T.; Baker, R. \& Dunbar, G. C. (1998). The Mini-International Neuropsychiatric Interview (MINI): The Development and Validation of a Structured Diagnostic Psychiatric Interview for DSM-IV and ICD-10. Journal of Clinical Psychiatry, 59(20), 22-33.

Sherwood, S. J. (1999). Relationship between childhood hypnagogic/ hypnopompic and sleep experiences, childhood fantasy proneness, and anomalous experiences and beliefs: An exploratory WWW survey. 
Journal of the American Society for Psychical Research, 93, 167-197.

Slade, P. (1976). An investigation of psychological factors involved in the predisposition to auditory hallucinations. Psychological Medicine, 6, 123-132.

Spanos, N. P.; Cross, P. A.; Dickson, K. \& Dubreuil, S. C. (1993). Close encounters: An examination of UFO experiences. Journal of Abnormal Psychology, 102, 624-32.

Tellegen, A. \& Atkinson, G. (1974). Openness to absorbing and selfaltering experiences ('absorption'), a trait related to hypnotic, suscepti- bility. Journal of Abnormal Psychology, 83, 268-277.

Thalbourne, M. (1982). A Glossary of Parapsychology. Londres: Heinemann.

Wallace, A. F. C. (1959). Cultural determinants of response to hallucinatory experience. Archives General of Psychiatry, 1, 58-59.

Wilson, S. C. \& Barber, T.X. (1983). The fantasy-prone personality: Implications for understanding imagery, hypnosis, and parapsychological phenomena. En A. A. Sheik (Ed.). Imagery: Current theory, research, and applications (pp. 340-387). Nueva York, NY: Wiley. 